\title{
The chromosomes of stomach cancers and myelogenous leucemias in comparison with normal human complex
}

\author{
Isamu Awano and Fukumi Tud.1 \\ Dept. Internal Medicine, Fukushima Medical College, Fukushima
}

Received May 18, 1959.

Recent investigations on the chromosomes of transplantable animal tumors have contributed strikingly to the understanding of the genetic pattern of tumors and the mechanism of malignant growth. A large amount of new data on the chromosomes in tumors has been collected during the past several years through the work dealing essentially with ascites tumors of rats and mice (cf. Makino 1957a). The results of those investigations have established the facts that the chromosome patterns of tumors generally differ from that of the normal tissue of the host species, and that the most commonly occurring tumor cells have characteristic chromosome-number modes together with particular chromosome constitutions and form a stem-cell lineage (or lineages) the members of which contribute respectively as the prime progenitors to the growth of each tumor (Makino $1957 \mathrm{a}, \mathrm{b}$, Makino \& Sasaki 1958). Accurate knowledge on the chromosomes in human tumors is rather meagre at present. It seems to be of real importance to consider the chromosome conditions in human tumors in relation to the type and property of those tumors.

Recently, the present authors have studied the chromosomes in tumor cells of four stomach cancers and four myelogenous leucemias. The tumor cells suspended in the ascites and bone-marrow taken from each patient were prepared according to the acetic orcein squash method for microscopical observations. In addition, the normal somatic chromosomes in bone-marrow cells of man were investigated, in order to compare the normal complex with the chromosome conditions in malignant cells.

Major conclusion derived from the present study was that each tumor possesses a stemline (or -lines) of tumor cells which are the principal progenitors of neoplasms. In each tumor the stem-cells are characterized by their own characteristic chromosome idiograms differing from those of normal tissues and by particular chromsomenumber modes. Particularly noticeable are the facts that these tumor cells show a complete lack of the $\mathrm{X}$-chromosomes, and that there is a definite increase in number of large V-shaped chromosomes in most tumors here studied.

The results of the present investigation are summarized as follows:- 




Figs. 1.3. Photomicrographs of somatic human chromosomes in vivo (bone-marrow). Fig. 1: Female. Figs. 2 and 3 : Male.

Table 1. Distribution of chromosome numbers in human somatic, gastric cancer, and leukemic cells observed in vivo

\begin{tabular}{|c|c|c|c|c|c|c|c|c|c|c|c|c|c|c|c|c|}
\hline Chromosome number & 363 & $3 x^{\prime}$ & 3940 & 41 & 42 & 43 & 44 & 45 & 46 & 47 & 48 & 49 & 50 & 51 & 91 . & Total \\
\hline Somatic cell & 1 & & 1 & 2 & 3 & 2 & 14 & 6 & 43 & $2 !$ & 6 & 0 & 2 & & & 82 \\
\hline  & & 1 & & 1 & & 1 & $\begin{array}{l}6 \\
1\end{array}$ & $\begin{array}{l}2 \\
1\end{array}$ & $\begin{array}{l}2 \\
2\end{array}$ & 2 & 5 & 6 & 2 & 3 & & $\begin{array}{l}14 \\
22\end{array}$ \\
\hline Leukemic cell & 1 & & 5 & 3 & 10 & 2 & 28 & 22 & 31 & 3 & 1 & 1 & & & 1 & 108 \\
\hline
\end{tabular}

1). It was shown on the basis of the study of normal bone-marrow cells that the number of chromosomes in the somatic human cell is determined as 46 in both sexes, though there is a variation ranging from 36 to 50 (Figs. 1-3, 6, 7, Table 1). The result of the present study is in agreement with the findings 1 jio of and Levan (1956), Ford and Hamerton (1956) and Makino and Sasaki (1955). There were found a few cells which show high polyploid numbers and polypolar divisions (Figs. 4-5). As shown in Figure 6, the idiogram analysis reveals that the normal somatic human complex is aligned into 22 pairs of homologous chromosomes 

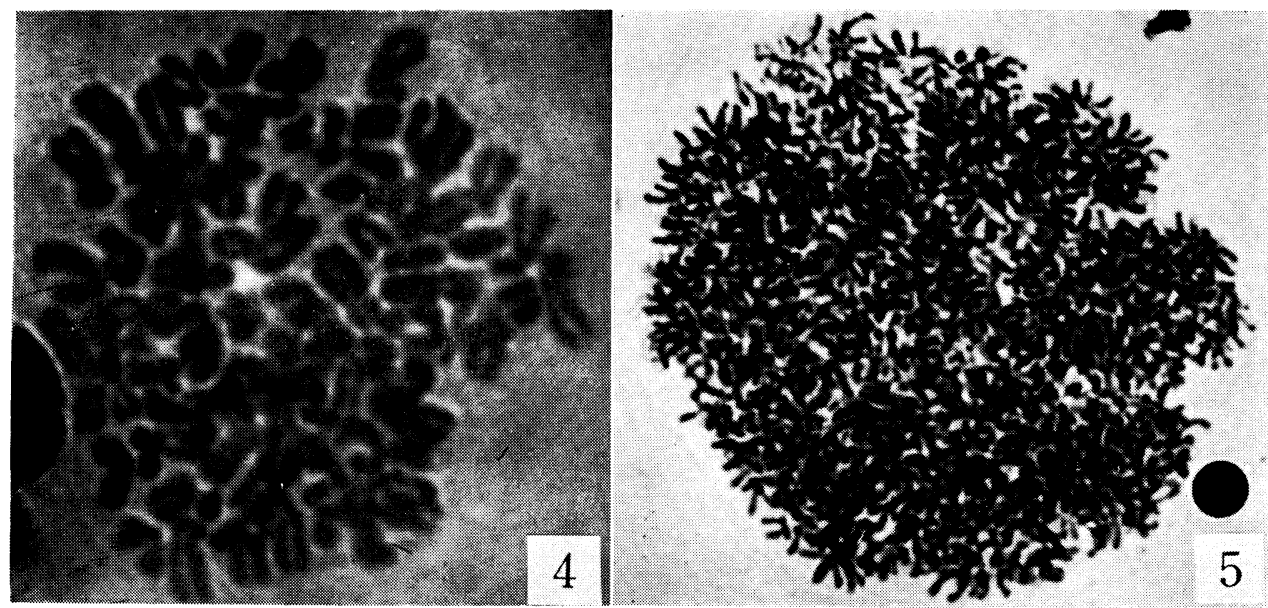

Figs. 4 5. Photomicrographs of somatic human chromosomes in bone marrow cells, showing a high polyploid complex in each.

and one pair of particular elements which are unequal in size in male cells, but equal in female cells. It is then evident that these two are the sex-determining elements: one of the unequal sized elements is represented by a chromosome of the large L-shape having a submedian centromere, while the other is a small submedian element. It is very likely that the larger one represents the $\mathrm{X}$-element while the smaller one is the Y. It was found that the 22 autosomal pairs consisted of one large pair of median V-shaped chromosomes, one large pair of submedian L-shaped ones, five large pairs of subterminal J-shaped ones, three pairs of medium L-shaped ones, six pairs of medium $\mathrm{J}$ - and small $\mathrm{L}$-shaped ones $(8 \mathrm{~J}$ - and 4 L-shaped ones) and six medium- and small-sized pairs of subterminal or terminal rod-shaped ones (12 rod-shaped ones) (Fig. 6).

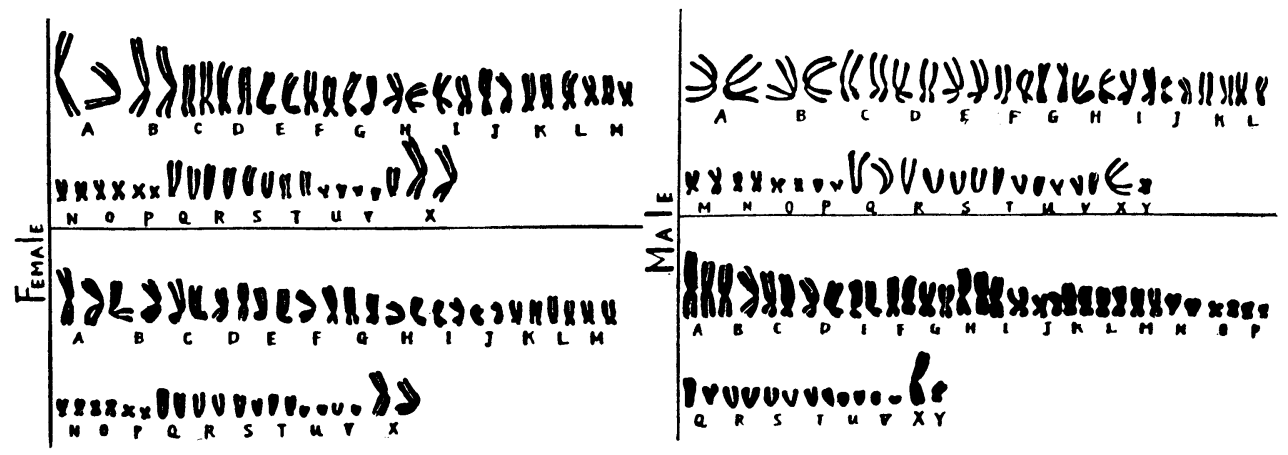

Fig. 6. Idiogram analyses of somatic human chromosomes in vivo.

2). The variation in number of chromosomes was observed in malignant cells from four gastric cancers and from four myelogenous leucemias, by way of comparison with that in the normal somatic chromosomes. The results indicate that the variation of the chromsome number is considerably larger in range in 
malignant cells than in normal cells. Though the number of chromosomes shows a pronounced variation in each tumor, there is always present a population of tumor cells that are characterized by a high frequency of occurrence and by a particular chromosome-number different frcm that of the normal tissue. Based on the conclusions from study of rat and mouse ascites tumors (cf. Makino 1957a), it is reasonable to assume that the most frequent tumor cells having a characteristic number-mode in human tumors here considered may represent the stemline cells which are the contributors to the growth of tumors. The above data are collected in Table 1 and further illustrated in Fig. 7.

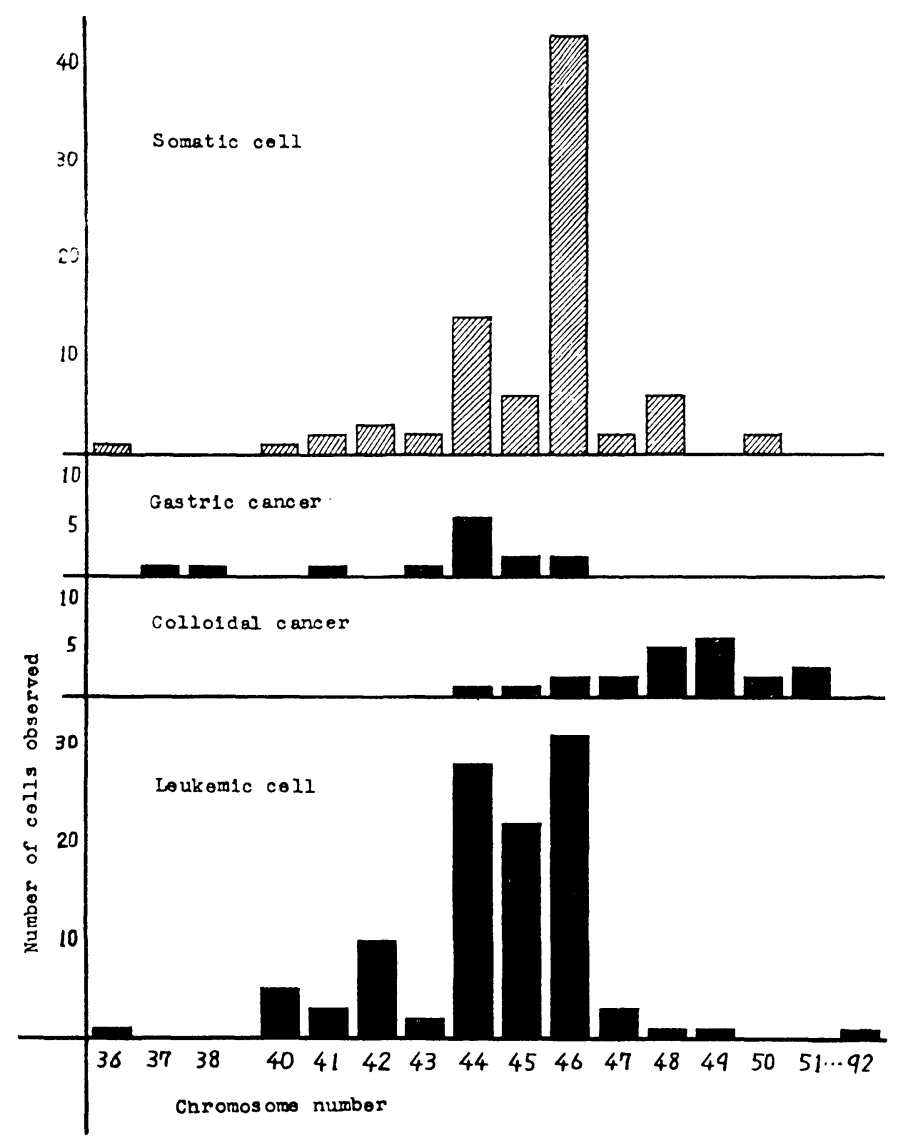

Fig. 7. Diagram showing the distribution of chromosome numbers. based on the data of Table 1 .

So far as the scope of the present study is concerned, the stemline chromosome number in the human tumors under study approximates the diploid number of man. For instance, the number is hypodiploid in an adeno-carcinoma, and hyperdiploid in a colloidal cancer, while in certain other tumors it is identical with the normal number.

3). In addition to the numerical change, the chromosome idiograms of human 
tumors here studied differ considerably from the normal idiogram. In other words, each tumor possesses its own characteristic chromosome idiogram. In most tumors considered here, an increase in number of large- $\mathrm{V}$-shaped chromsomes is noticeable. For example, the st $\in \mathrm{m}$-cells of two adeno-carcinomas are characterized by containing

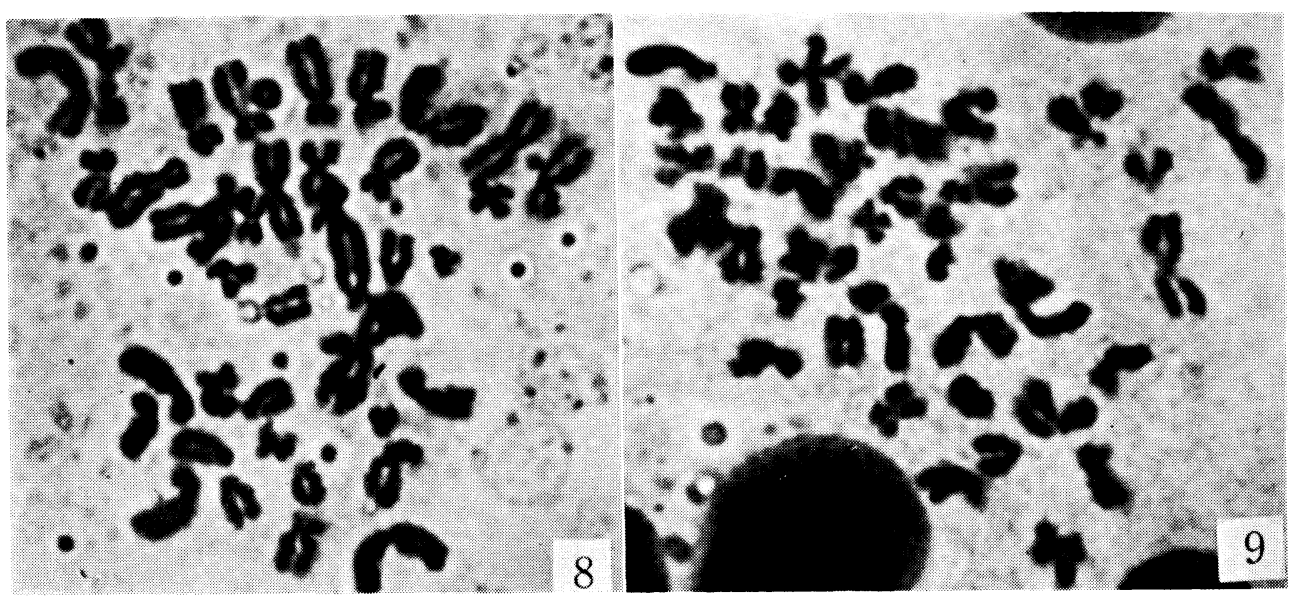

Figs. 8-9. Chromosome complexes of human cancer cells in ascites. Fig. 8: Adenocarcinoma. Fig. 9 : Colloid cancer.
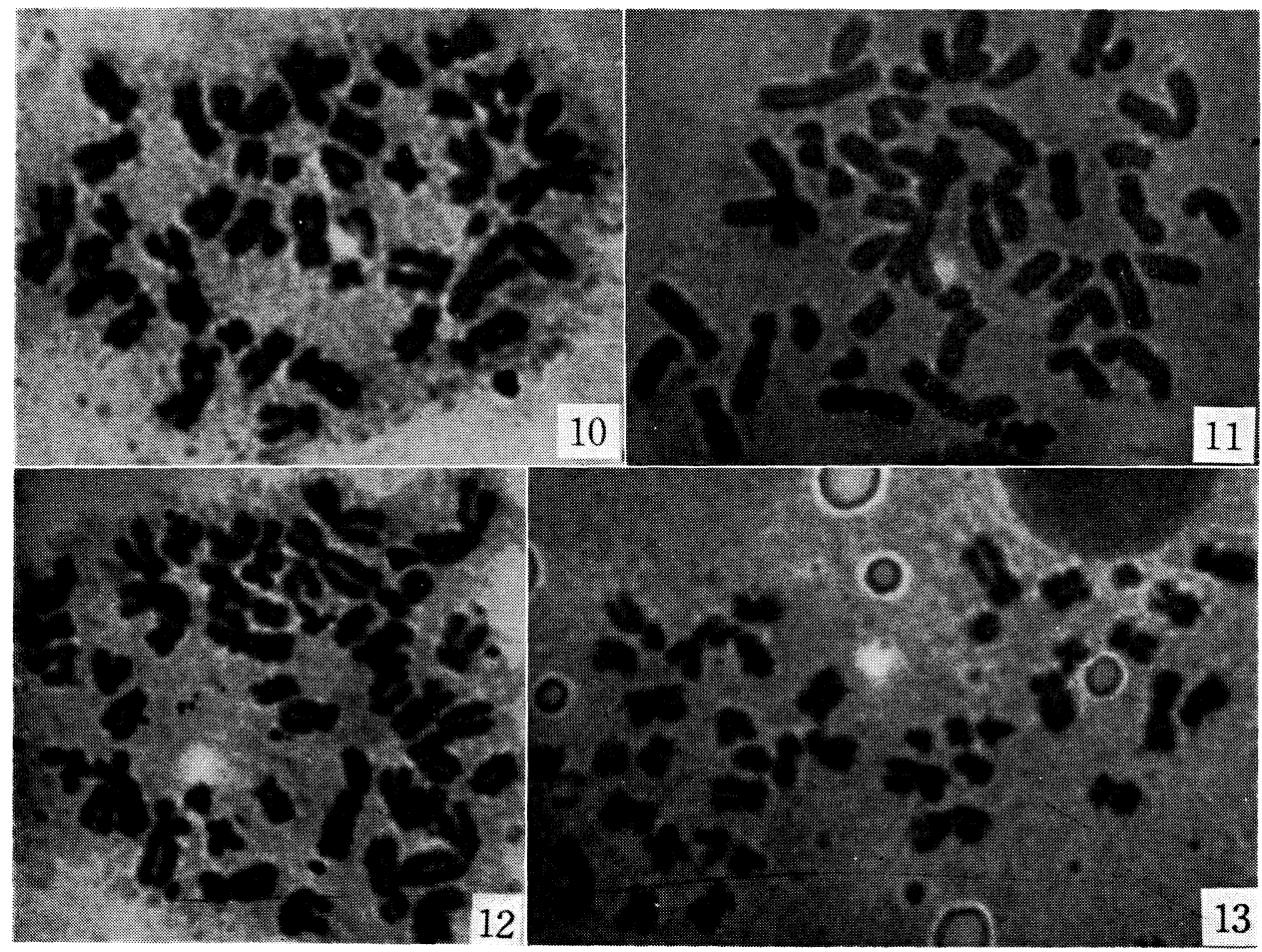

Figs. 10-13. Chromosome complexes of human myelogenous leucemic cells. Figs. 10-11: Female. Figs. 12-13: Male. 
4 large V-shaped chromsomes, while those of a colloidal cancer are marked by the presence of $3 \mathrm{~V}$-shaped ones (Figs. 8, 9, 14).

In contrast to the above cases, the stem-cells of the myelogenous leucemias show 44-46 chromosomes which are nearly identical both in number and morphological features to the somatic complex (Figs. 10-13, 15). It is most probable that the similarity of chromsomes may be superficial, and that the chromosomes of the leucemic cells may undergo changes in their inner structure.

4). Detailed comparison of the chromosomes between the normal cell and neoplastic cells has revealed that a large L-shaped chromosome corresponding to the



Fig.14. Idiogram analyses in human cancers in vivo.

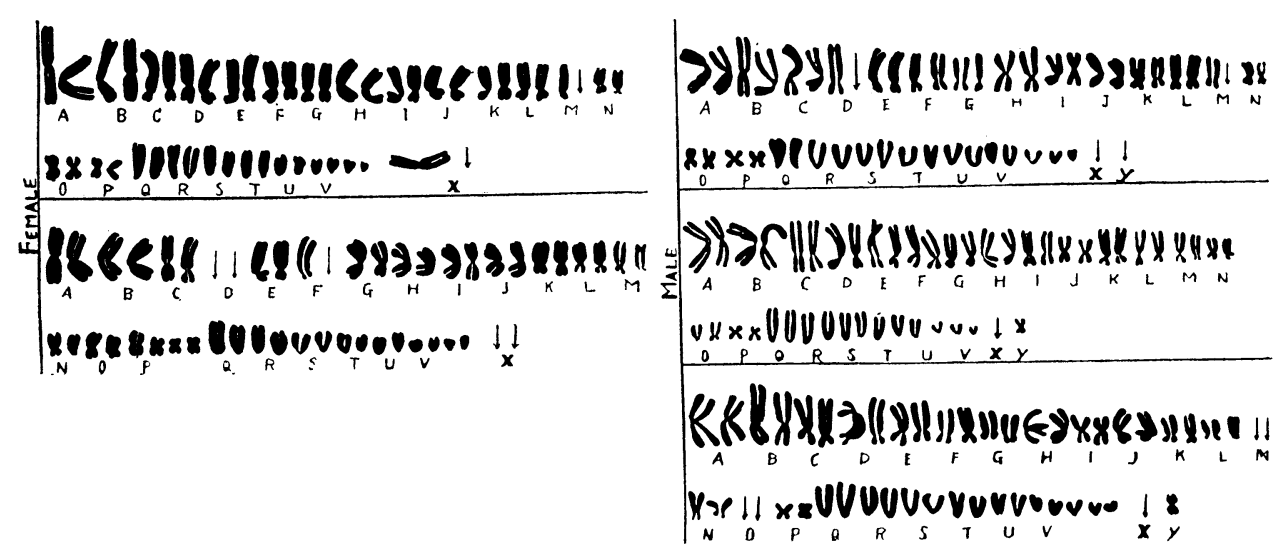

Fig. 15. Idiogram analyses in human myelogenous leucemias in vivo.

$\mathrm{X}$-element is absent in most tumor cells of the gastric cancers and myelogenous leucemias here investigated (Figs. 14-15). Namely, there are in tumor cells a lack or an addition of certain chromosomes by which evidence the tumor cells are distinguishable from the normal somatic cell.

It is a pleasure to record here a debt of gratitude to Professor Sajiro Makino for his kindness in improving the original manuscript.

\section{References}

Ford, C.E. and Hamerton, T.L. 1956. Nature 178 : 1020-1023.

Makino, S. 1957a. The chromosome cytology of the ascites tumors of rats, etc. Intern. Review of Cytology, VI : 25-84.

1957b. Proc. Intern. Genet. Symp., 1956 : 177-181.

Makino, S. and Sasaki, M. 1958. J. Nat. Cancer Inst. 20 : 465-487.

and 1959. Proc. Jap. Acad. $35: 99-104$.

Tjio, J.H. and Levan, A. 1956. Hereditas $42: 1-6$. 